\title{
Automated Blood Cells Extraction and Counting
}

Bilal M. Zahran ${ }^{1}$, Belal A. Ayyoub ${ }^{1}$, Rushdi S. Abu Zunait ${ }^{1}$, Ahmad A. Sharadqeh ${ }^{1}$

${ }^{1}$ Al-Balqaa Applied University / Faculty of Engineering Technology

Computer Engineering Department

\section{ABSTRACT}

zahranb@bau.edu.jo

Automated blood cells extraction and counting in medical images is an important and sometimes necessary procedure in medical diagnostics and treatment. In this paper an automated blood cells extraction and counting method from microscopic images was proposed. The method is based on morphological filtering. The proposed method was tested using different background noises, resolutions and different colored images. The results are encouraging and the method gave an excellent performance for white blood cells and acceptable performance for red blood cells.

\section{Keywords}

Red blood cell (RBC) counting, White blood cell (WBC) counting, morphological filtering, RBCs classification, WBCs classification

\section{Council for Innovative Research}

Peer Review Research Publishing System

\section{Journal: INTERNATIONAL JOURNAL OF COMPUTERS \& TECHNOLOGY}

Vol 12, No. 9

editor@cirworld.com 


\section{INTRODUCTION}

Blood is a connective tissue consisting of cells suspended in plasma. Blood's major functions are to transport various agents such as oxygen, carbon dioxide, nutrients, wastes, and hormones. Blood cells are composed of erythrocytes (red blood cells, RBCs), leukocytes (white blood cells, WBCs) and thrombocytes (platelets). RBCs' two principal functions are to move oxygen from lung to tissues elsewhere and transport carbon dioxide from tissues to the lung. Whereas, the Leukocytes or white blood cells are part of the immune system [1]. The conventional device used to count blood cells is the hemocytometer. It consists of a thick glass microscope slide with a rectangular indentation creating a chamber of certain dimensions. This chamber is etched with a grid of perpendicular lines. It is possible to count the chamber of cells in a specific volume of fluid, and calculate the concentration of cells in the fluid [2,3]. To count blood cell, physician must view hemocytometer through a microscope and count blood cells using hand tally counter. Normally, the counting task is time-consuming and laborious.Many commercially available products have been developed to automatically count RBCs or WBCs [8]. Their advantages include automatic cell counting cells without hand tally counter, no requirement of messy washing and no associated biohazard. However, these products are expensive. Several attempts have been made to mimic the procedure of cell recognition from image. The major application of neural networks was devoted to the WBCs classification via extracted morphologic parameters [4,5,12 and 13].

\section{THE PROPOSED BLOOD CELLS CLASSIFICATION COUNTING ALGORITHM}

We presented a morphological filtering baesd method to classify and count RBCs and WBCs. The proposed algorithm is illustrated in Figure 1.

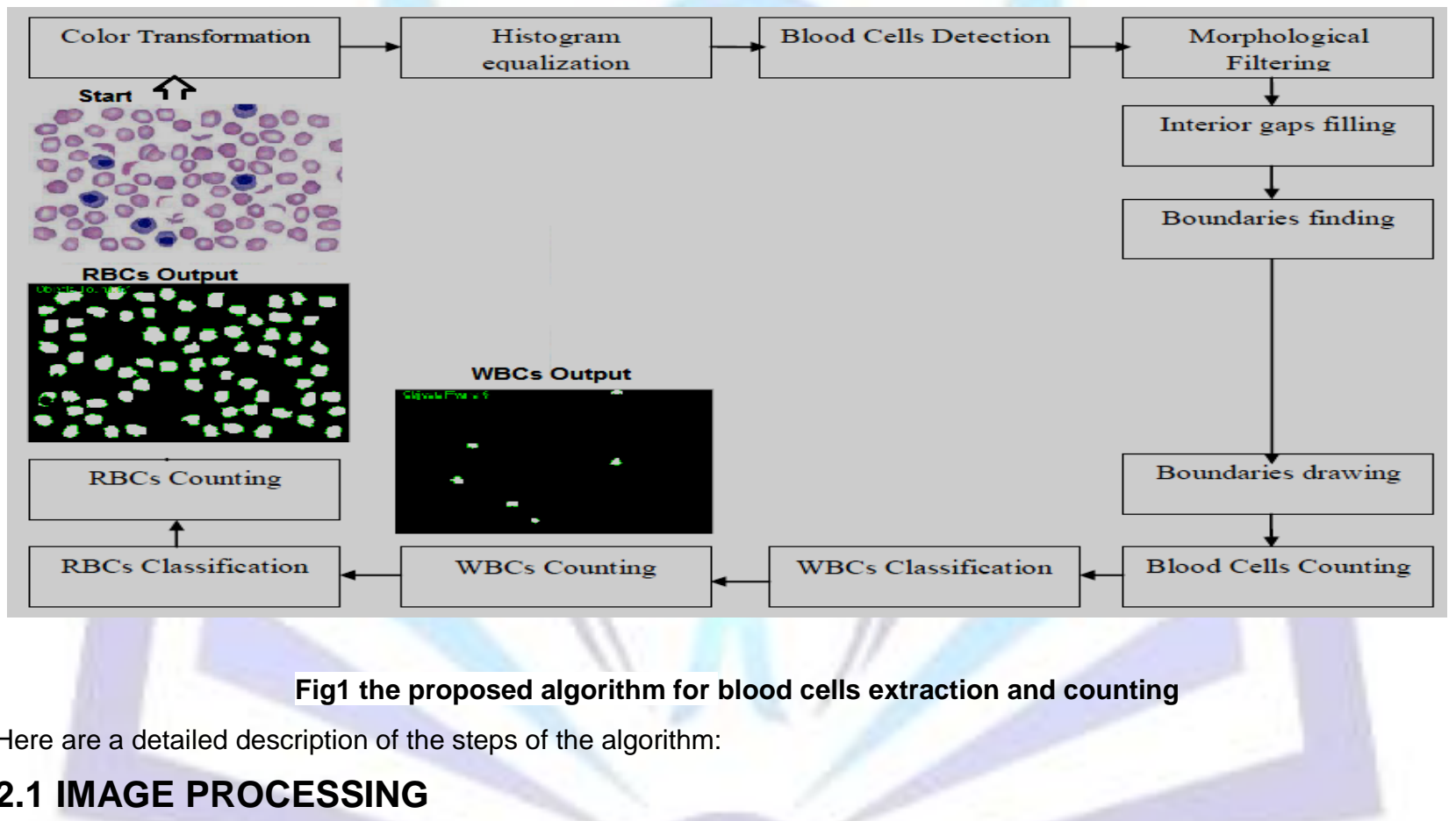

The main image processing tasks consists of enhancing the image's qualities and deleting overlapped blood cells in the boundary area of the image. Both tasks can be subdivided into smaller tasks here we are illustrating each step in the next subsections.

\subsubsection{Color Transformation}

To change the colored image to a gray image we should eliminate the hue and saturation values from the original image but keeping the luminance value which correspond to the gray image. After converting the colored image to gray image, we converted the gray image to binary image. The output image of this process replaces all pixels in the input image with luminance greater than level with the value 1 (white) and replaces all other pixels with the value 0 (black).

\subsubsection{Histogram equalization}

This process adjusts intensity values of the image by performing histogram equalization involving intensity transformation.

\subsubsection{Blood Cells Detection}

The objective of blood cell detection is to detect cells which differentiate themselves from the background in terms of contrast; we applied gradient based edge detection Canny technique. The canny method is applied to search for edges by looking for local maxima of the gradient. We chose two threshold values, to detect strong and weak edges, and includes 
the weak edges in the output only if they are connected to the strong edges. The Canny Edge Detected image is shown in Figure 2.a

\subsection{MORPHOLOGICAL FILTERING}

We applied some morphological filtering to remove noise from an imperfect segmentation and to get smooth region boundaries for shape analysis:

\subsubsection{Dilation}

Applying morphological operator to create a structuring element specified by interested shapes, disk-shaped structuring element. The dilated image is shown in Figure 2.b

\subsubsection{Interior gaps filling}

The dilated gradient mask shows the outline of the blood cell quite nicely, but there are still holes in the interior of the cell. Filling internal holds of the connected element get the biggest area in the processed image. All Blood Cells were filled except overlapping cells (which locate on borders of the image) because they are uncompleted cells (unenclosed objects) The Filled cells image is shown in Figure 2.c.

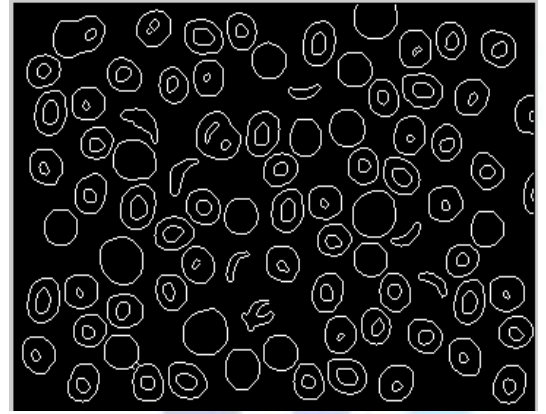

(a)

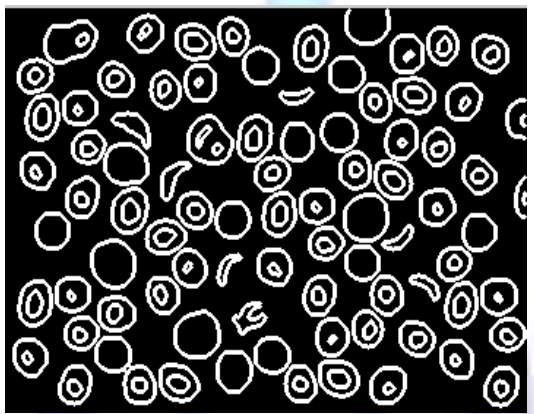

(b)

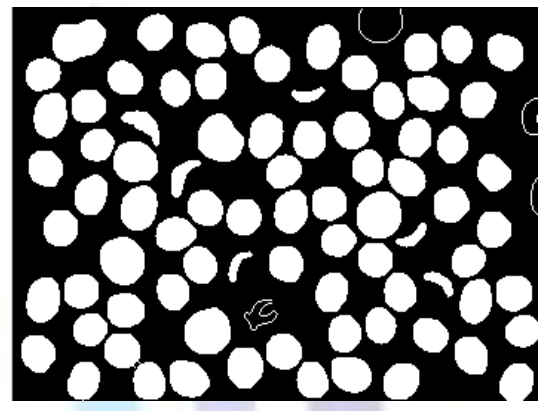

(c)

Fig 2 Morphological filtering: (a) Canny Edge Detected image, (b) Dilated Cells image, (c) Filled cells image

\subsection{EROSION (OBJECTS SMOOTHING)}

All of Blood Cells have been successfully segmented. But we will cancel the overlapping cells with borders (Border padding). Finally, in order to make the segmented object look better, the objects in the processed image can be smoothed by eroding the image

\subsubsection{Border padding}

Removing object connected to borders as the neighborhood operator block slides over the entire image, some of the pixels around the border may be missing, if the center pixel is on the border of the image. The missing pixels will be padded using 0 values (black) to complete the image, see Figure 3.

\subsubsection{Thresholding}

The WBCs can be identified base on the blue level in the RGB (multidimensional matrix). The obtained mean values will be used to classify WBCs from other blood cells. The RBCs can be identified base on the red level in the RGB (multidimensional matrix).

This step is worked after the gray image obtained,if the value of the pixel less than or equal to the threshold value it will be 
considered as a background pixel, if the pixel value is higher than the threshold value it will be considered as an object value:

$$
\mathrm{g}(x, y)= \begin{cases}1, & \mathrm{f}(x, y)>\text { threshold } \\ 0, & \mathrm{f}(x, y) \leq \text { threshold }\end{cases}
$$

This where $g(x, y)$ is the threshold image, $f(x, y)$ is the original image, and $x, y$ are the pixel coordinate in the image .l.e. pixel labeled 1 correspond to objects (foreground) pixel labeled 0 correspond to the background.

\subsubsection{Automatically Blood Cells Counting}

In this stage we will count all of BCs in the previous image (without any classification into RBCs, WBCs or SBCs) with two main steps:

1. Finding the boundaries of blood cells.

2. Drawing the boundaries of blood cells.

Before Interior gaps filling we notice that we got (164) blood cells (see Figure 4.a) but, the actual result was (88), so we will apply filling holes function to get more accuracy as the Figure 4.b shown:

(a)
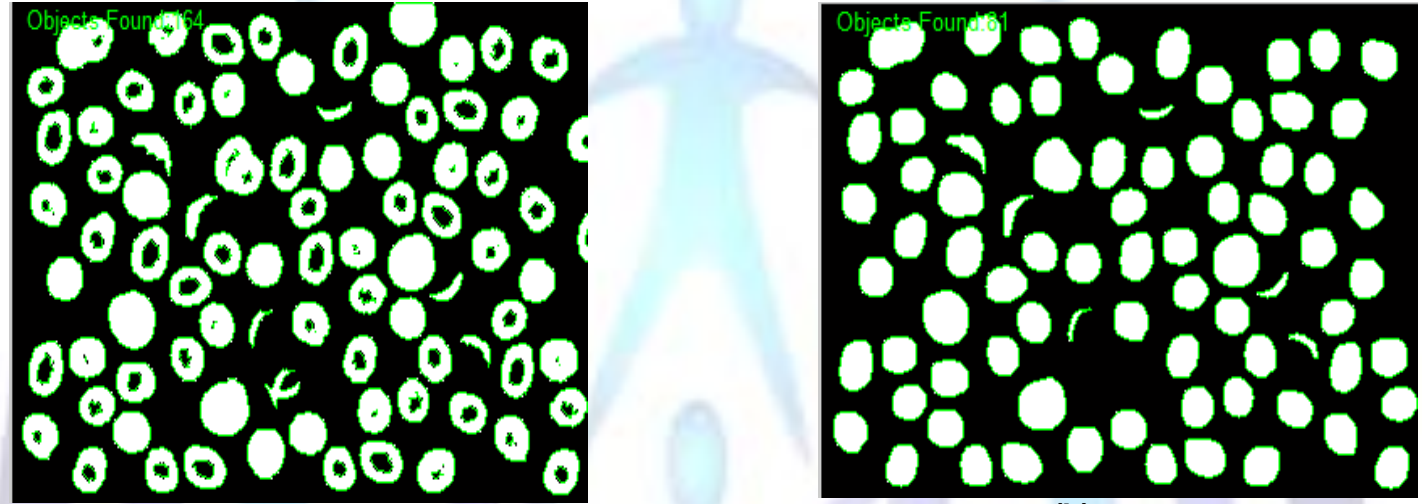

(b)

Fig 4 (a) the result of counting (without filling), (b) the result of counting with filling

After Interior gaps filling we notice that we got (81) blood cells from (88) actual count. The (81) BCs are (67 RBCs, 0 overlapping RBCs, 2 overlapped RBCs, 5 WBCs, 1 overlapping WBCs, 6 SBCs, 0 overlapped SBCs) From actual (68 RBCs, 2 overlapping RBCs, 4 overlapped RBCs ,5 WBCs, 1 overlapping WBCs, 6 SBCs, 2 overlapped SBCs).

\subsection{BLOOD CELLS CLASSIFICATION AND COUNTING}

Patterns of blood cells are classified based on their similarity to each other based on general features like color (as we applied) which help us to extract same type of blood cells then count it for the purposes of disease determination.

The images were analyzed by manually looking for blood cells. After that, the blood cells were counted using the proposed blood cell counting method, automatically.

The second experiment system was done on many microscopic (Blood smear) images which are different in (number of BCs, resolution, and colors scale of BCs and backgrounds). We will explain these steps in the following two subsections: 


\subsubsection{WBCs Extraction and counting}

The first experiment was conducted on a set of microscopic images to extract and count white blood cells and the main steps shown in Figure 5:
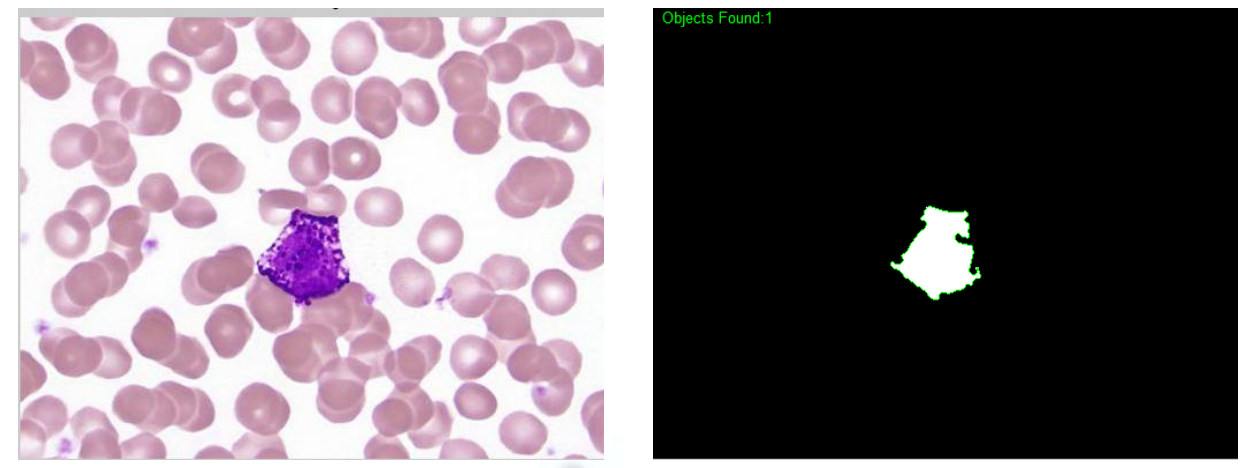

Fig 5 WBCs count in a microscopic image

\subsubsection{RBCs Extraction and counting}

The Second experiment was conducted on the set of microscopic images to extract and count red blood cells and the main steps shown as:
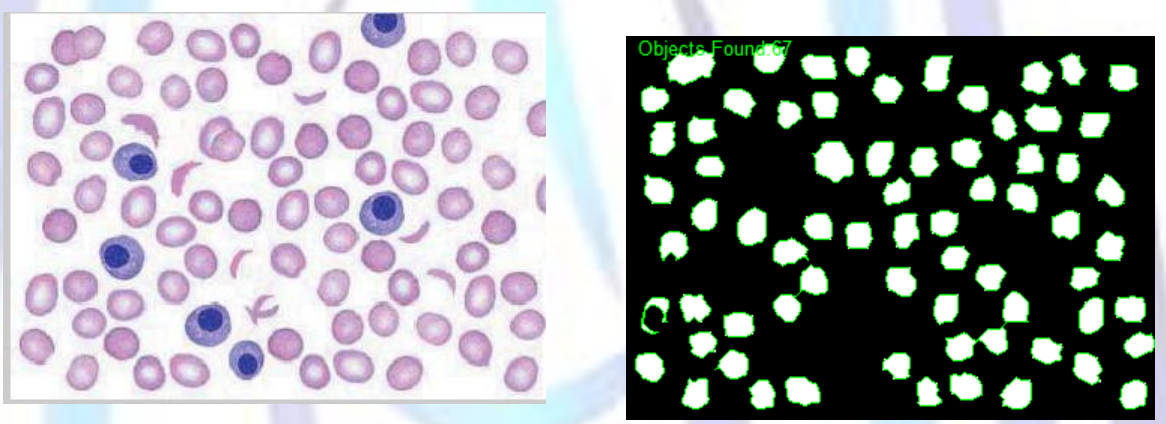

Fig 6 RBCs count of (original) image

\section{EXPERIMENTAL RESULTS}

The experiments for the system were done on many microscopic (Blood smear) images that taken from low resolution camera with different number of BCs, colors scale and backgrounds, the software that controls the image processing and implements our system are MATLAB R2008b. We applied our method to 16 microscopic images (experimental counts) then compared it to the actual ones (actual counts) as shown in the Table 1:

Table 1. The results of 16 experiments

\begin{tabular}{|c|c|c|c|c|c|c|c|c|}
\hline \# & image name & Act. WBCs & Exp. WBCs & Act. RBCs & Exp. RBCs & Act. Overlapping RBCs & Act. Overlapped RBCs & Total Act. RBCs \\
\hline 1 & dd3 & 1 & 1 & 9 & 35 & 5 & 112 & 126 \\
\hline 2 & dd4 & 1 & 1 & 27 & 56 & 11 & 40 & 78 \\
\hline 4 & $\mathrm{rr}$ & 2 & 2 & 390 & 467 & 45 & 101 & 536 \\
\hline 5 & kk & 7 & 9 & 4 & 6 & 9 & 130 & 143 \\
\hline 6 & oo & 2 & 3 & 164 & 384 & 36 & 35 & 235 \\
\hline 7 & img02 & 2 & 2 & 415 & 176 & 24 & 215 & 415 \\
\hline 8 & $\mathrm{~mm}$ & 6 & 5 & 57 & 15 & 15 & 52 & 124 \\
\hline 9 & $\mathrm{nn}$ & 1 & 1 & 69 & 4 & 15 & 16 & 100 \\
\hline 10 & dd1 & 1 & 2 & 13 & 42 & 34 & 77 & 124 \\
\hline 11 & $\mathrm{dd} 2$ & 2 & 2 & 26 & 45 & 19 & 32 & 77 \\
\hline 12 & microl & 2 & 2 & 16 & 30 & 17 & 22 & 55 \\
\hline 14 & ee & 1 & 0 & 71 & 81 & 18 & 6 & 95 \\
\hline 15 & $\mathrm{tt}$ & 5 & 0 & 44 & 45 & 25 & 4 & 109 \\
\hline 16 & original & 5 & 5 & 68 & 69 & 2 & 4 & 74 \\
\hline
\end{tabular}


The results in Table 1 shows that the proposed method counts WBCs in an efficient manner, while counts the RBCs in a less efficient manner compared with the acual results. The reason is that the proposed method handles the overlapped groups of BCs as a single element.

\section{CONCLUSION}

In our proposed approach, a methodology for automated blood cells extraction, classification and counting which uses morphological filtering was introduced. Furthermore, this work shows the problems that faced applying of automatic cells recognition in bright field microscopy [11], it is a difficult task due to the immense variability of cells appearance. The algorithm cannot count the exact number of the blood cells when these are overlapped with each other. So, the algorithm considers the overlapped groups of $\mathrm{BCs}$ as a single element. The quality of the results is closely related to three parameters: thresholds for the Binarization and segmentation, distribution of the gray levels intensity and the strength of the response of the morphological operators. We can improve the results varying these three parameters. However, the selection of appropriate features is critical for extracting and classification BCs. After many experiments on different backgrounds, resolutions and color scale microscopic images, our system gave good results for blood cells extraction, classification, and counting which achieve an excellent performance for WBCs while it was acceptable for RBCs.

\section{REFERENCES}

[1] K.S. Saladin, Anatomy and Physiology: The unity of form and function, McGraw-Hill, NY, 4th, chap. 18, pp. 680-696, 2007.Ding, W. and Marchionini, G. 1997 A Study on Video Browsing Strategies. Technical Report. University of Maryland at College Park.

[2] J.P. Mather and P.E. Roberts, Introduction to cell and tissue culture: Theory and Technique, Plenum Press, NY and London, 1998.

[3] D.R. Caprette, "Introduction Laboratory : using a counting chamber," Bios211, 2007

[4] Q. Zheng, B.K. Milthorpe, and A.S. Jones, "Direct neural network application for automated cell recognition," Cytometry 57A, pp. 1-9, 2004

[5] T. Markiewidz and S. Osowski, "Data mining techniques for feature selection in blood cell recognition," in Proc. ESANN. Symp. Bruges, BG, pp. 407-412, April 2006.

[6] F. Scotti, "Automatic Morphological Analysis for Acute Leukemia Identification in Peripheral Blood Microscope Images," in Proc. IEEE Int. Symp. CIMSA, Giardini Naxos, Italy, pp. 96-101, July 2005.

[7] W. Wongseree and N. Chaiyaratana, "Thalassemic Patient Classification Using a Neural Network and Genetic Programming," IEEE, pp. 2926-2931, 2003.

[8] Effector Cell Institute, Inc, “CYTOREONTM," news, Tokyo, Japan, December 2007.

[9] D. Meyer, J. Denzler \& H. Niemann, (1998). Model based extraction of articulated objects in image sequences for gait analysis, Proceeding of the IEEE Int. Conf. on Image Processing, pp. 78-81.

[10] F.Sadeghian, Z.Seman, A. RahmanRamli, B.HishamAbdul Kahar, and M-lqbalSaripan(2009), "A Framework for White Blood Cell Segmentation in Microscopic Blood Images Using Digital Image Processing", Department of Computer and Communication Systems Engineering, University of Putra, Malaysia.

[11] X. Long, W. L. Cleveland, Y.L. Yao (2005) "Effective automatic recognition of cultured cells in bright field images using fisher's linear discriminate preprocessing", Columbia University, USA.

[12] M. Veluchamy, K. Perumal and T. Ponuchamy Feature Extraction and Classification of Blood Cells Using Artificial Neural Network. ajassp.2012. V9. pp:615-619

[13] N. Theera-Umpon and P. Gader. System-Level Training of Neural Networks for Counting White Blood Cells. IEEE TRANSACTIONS ON SYSTEMS, MAN, AND CYBERNETICS—PART C: APPLICATIONS AND REVIEWS, V. 32, NO. 1, Feb. 2002 